\title{
$\underline{P-129}$
}

\section{Stevioside Potentiates Insulin Sensitivity by Elevating Insulin-Stimulated Glucose Uptake in 3T3-L1 Adipocytes}

\author{
Nabilatul Hani Mohd Radzman ${ }^{1, *}$, Zainah Adam ${ }^{2}$, Siti Safura Jaapar ${ }^{1}$, Aishah Adam ${ }^{1}$ and \\ Wan Iryani Wan Ismail ${ }^{1}$ \\ ${ }^{1}$ Faculty of Pharmacy, UiTM Puncak Alam, 42300 Bandar Puncak Alam, Selangor Darul Ehsan, Malaysia; ${ }^{2}$ Medical \\ Technology Division, Nuclear Malaysia, Bangi, 43000 Kajang, Selangor Darul Ehsan, Malaysia; \\ E-mail:nabilaradzman@gmail.com
}

Stevia rebaudiana Bertoni is a herb common to tropical and subtropical regions particularly Asia and South America. In countries like Malaysia, the sweet leaves of this plant have been used in daily cooking and as a form of alternative therapy by communities practicing folk medicines. The zero-calorie value of stevia leaves also provide a beneficial sweetening alternative to patients with metabolic syndromes like diabetes and obesity. A cell culture model of 3T3-L1 preadipocytes was implemented in this study to investigate the effects of stevioside, a major component extracted from the Stevia rebaudiana Bertoni plant on improving insulin sensitivity. 3T3-L1 preadipocytes were first differentiated to mature adipocytes prior to tests. The differentiation process was later confirmed by performing Oil-Red-O staining. The degree of insulin sensitivity was measured by performing counts via the radioactive 2-deoxyglucose uptake assay. A separate assay was done to estimate the optimum insulin concentration in the glucose uptake of the 3T3-L1 adipocytes. Cells treated with stevioside showed significant increase in glucose uptake at lower concentrations compared to a diabetic drug, rosiglitazone maleate at higher concentrations of the compound. The enhancement of glucose uptake was 2.34 -fold $(p<0.001)$ in the cells treated with $30 \mu \mathrm{M}$ stevioside. Stevioside was thus seen to elevate insulin-stimulated glucose uptake concomitant with potentially enhanced insulin signaling in the 3T3L1 cell culture model.

Keywords: Stevia rebaudiana, stevioside, insulin, 2-deoxyglucose, glucose uptake, rosiglitazone. 